\title{
Neonataler Aszites: Mekoniumileus mit Perforation bei Mukoviszidose
}

\author{
S. Stoll-Becker ${ }^{1}$, I. Reiss ${ }^{1}$, G. Hüls ${ }^{1}$, A. Hauch², M. Hermsteiner ${ }^{3}$, L. Gortner ${ }^{1}$ \\ 1 Zentrum für Kinderheilkunde und Jugendmedizin, Justus-Liebig-Universität, Gießen \\ 2 Zentrum für Chirurgie, Justus-Liebig-Universität, Gießen \\ ${ }^{3}$ Zentrum für Frauenheilkunde, Justus-Liebig-Universität, Gießen
}

\section{Zusammenfassung}

Neonataler Aszites erfordert eine breite differenzialdiagnostische Abklärung. Es kommen kardiovaskuläre Ursachen, Fehlbildungen, Chromosomenanomalien, Infektionen, fetale Anämie, Tumoren, metabolische und mütterliche Ursachen in Betracht. In 30\% der Fälle lässt sich keine Ursache finden. Der Mekoniumileus stellt sich als eine mögliche gastrointestinal zugrunde liegende Erkrankung dar. Wir berichten über ein Frühgeborenes von 35 + 3 Schwangerschaftswochen mit kompliziertem Mekoniumileus bei Mukoviszidose.

\section{Neonatal Ascites: Meconiumileus with perforation secondary to mucoviszidosis}

Many conditions are known associated with neonatal ascites such as cardiac and vascular malformations, as well as malformations of the brain, kidney, lung and bone, chromosomal abnormalities, infections, fetal anemias, tumors, metabolic and maternal conditions. In $30 \%$ no reason can be found. Meconium ileus is a causal gastrointestinal abnormality. We report about a preterm infant 35 weeks of gestation with complicated meconium ileus because of mucoviscidosis.

\section{Fallbericht}

Anamnese: Aufnahme der Mutter (21-jährige G1/P1, kaukasischer Herkunft) in der Universitäts-Frauenklinik Gießen in der $35+3$ SSW bei zuvor unauffälligem Schwangerschaftsverlauf. In der pränatalen Ultraschalluntersuchung zeigte sich ausgeprägter neonataler Aszites (Abb.1), daher erfolgte die Sectio cesarea am gleichen Tag.

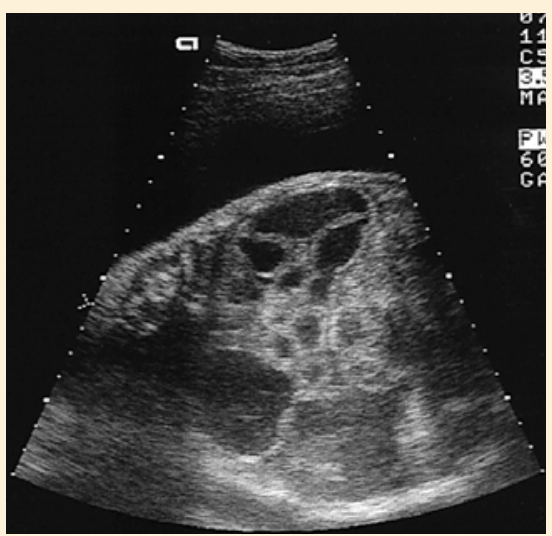

Abb. 1 Pränatale Ultraschalluntersuchung mit einem ausgeprägten neonatalen Aszites und dilatierten Darmschlingen als Hinweis auf eine intestinale Obstruktion.

Apgar 7/8/9, NSpH 7,23, Geburtsgewicht: $3100 \mathrm{~g}$. Die Intubation im Kreißsaal wurde nötig bei Ateminsuffizienz durch Zwerchfellhochstand bei massiv aufgetriebenem Abdomen (Abb. 2). Es konnte sehr viel galliges Fruchtwasser gastral abgesaugt werden. Nach Anlage eines arteriellen Zuganges, zentralen Venenkatheters, Volumengabe und Transfusion erfolgte die sofortige Operation. Abb. 3 zeigt den intraoperativen Situs.

Befunde: Die Befunde des kleinen Patienten sind in Tab. 1 dargestellt.

Diagnose: Komplizierter Mekoniumileus bei Mukoviszidose.

\section{Therapie und Verlauf}

Intraoperativ wurden etwa $700 \mathrm{ml}$ blutig mekoniumhaltiger Aszites abgesaugt. Es mussten $25 \mathrm{~cm}$ Dünndarm reseziert und ein doppelläufiges lleo-Jejunostoma angelegt werden. Eine Nahrungskarenz bis zum 8. postoperativen Tag war nötig, dann

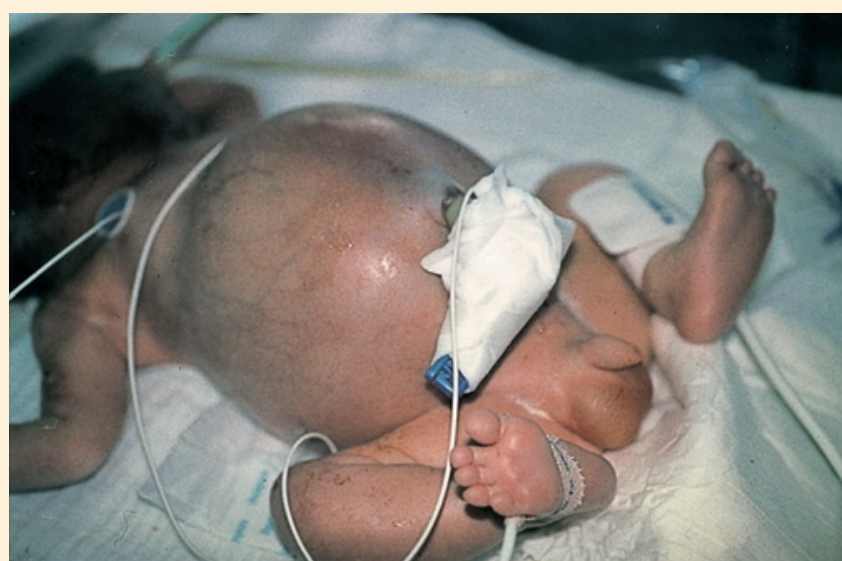

Abb. 2 Proportionen des betroffenen Patienten wenige Minuten postnatal mit massiv dilatiertem Abdomen, glänzender Bauchhaut und vermehrter Venenzeichnung.

erfolgte langsamer Nahrungsaufbau mit volladaptierter Formelernährung. Über die Magensonde, den aboralen Schenkel des Ileo-Jejunuostomas und rektal erfolgte Spülung mit $\mathrm{NaCl} 0,9 \%$ / Acetylcystein. Die Peritonitis wurde mit antibiotischer Therapie mit Gentamicin und Cefotaxim i.v. behandelt. Ab dem 12. postoperativen Tag wurde Chymus aus dem oralen Schenkel in

Tab.1 Diagnostik bei Frühgeborenem mit Mekoniumileus bei Mukoviszidose

\section{Befunde:}

is Histopathologie der Rektumsaugbiopsie: Kein Hinweis auf eine neuronale intestinale Dysplasie, kein Hinweis auf eine Innervationsstörung im Sinne eines Morbus Hirschsprung. Segmentale Lumenokklusion durch schleimiges Material.

s Neugeborenen-Screening: Pathologisch erhöhtes immunreaktives Trypsin mit $87 \mathrm{ng} / \mathrm{ml}$ bzw. bei Kontrolle $193 \mathrm{ng} / \mathrm{ml}$.

Elastase im Stuhl: Pathologisch erniedrigt mit $<100 \mu \mathrm{g} / \mathrm{g}$ Stuhl.

Chymotrypsin im Stuhl: Pathologisch erniedrigt mit $<1 \mathrm{U} / \mathrm{g}$ Stuhl.

is Schweißtest: Pathologisch erhöht mit $\mathrm{Cl}>100 \mathrm{mMol} / \mathrm{l}$.

ts Rektale Potenzialdifferenz-Messung: Aufgrund der anatomischen Verhältnisse technisch nicht möglich gewesen.

is Molekulargenetik: CFTR-Genort heterozygot für $\Delta \mathrm{F} 508$. 


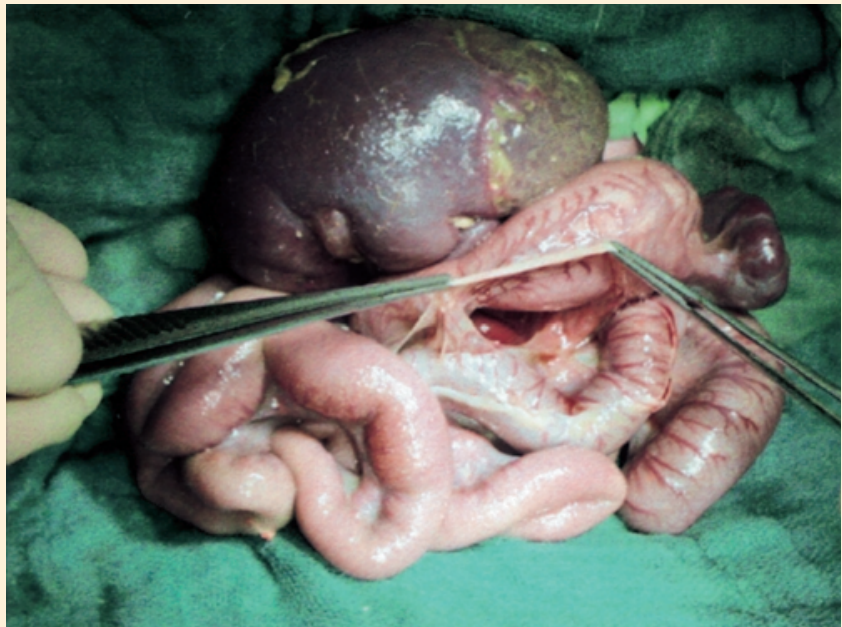

Abb. 3 Intraoperativer Situs: Der dilatierte prästenotische Jejunumlleumdarmanteil ist stark mit Mekonium gefüllt. Poststenotisch sieht man das enge Mikrokolon im Sinne eines Non-used-Colon. Es bestehen Verwachsungen zu der seitlichen Bauchwand und dem Omentum schon mit Gefäßeinsprossungen. Das terminale lleum ist perlschnurartig verengt. Durch einen Mesenterialeinriss hindurch sieht man stark torquiertes Dünndarmkonvolut mit Nekrosen und Perforation. Nach Eröffnung des stark entzündlich verdickten Peritoneums entleerte sich ca. $700 \mathrm{ml}$ blutig mekoniumhaltiger Aszites.

den aboralen Schenkel des lleo-Jejunostomas umgefüllt. Am 30. postoperativen Tag zeigte sich das klinische Bild eines Bridenileus mit Erbrechen. In einer Reoperation erfolgte die Lösung der Briden. Der anschließende weitere Nahrungsaufbau und das Gedeihen waren unproblematisch.

\section{Ätiologie, Epidemiologie und Pathogenese}

Die Mukoviszidose stellt bei Kaukasieren mit 1:2000-2500 die häufigste autosomal-rezessiv vererbbare Stoffwechselerkrankung dar. Jeder 20. bis 30. ist klinisch asymptomatischer heterozygoter Genträger. 10-15\% der Patienten mit Mukoviszidose manifestieren sich mit einem Mekoniumileus. 90\% der Neugeborenen mit Mekoniumileus haben als Grunderkrankung eine Mukoviszidose; eine neuronale intestinale Dysplasie muss ausgeschlossen werden.

Die Pathogenese der Mukoviszidose besteht in einer Dysfunktion des sekretorischen Epithels aller exkretorischen Drüsen, mit exokriner Pankreasinsuffizienz und polyglandulären Dyskrinie. Ab der 12.-15. Schwangerschaftswoche bildet sich beim Feten Mekonium, dieses haftet als zäh-visköses Mekonium ab der 20.25. Schwangerschaftswoche bei Feten mit Mukoviszidose der Darmwand des terminalen lleums so stark an, dass eine Obstruktion ausgelöst werden kann.

Die Mukoviszidose wird durch Mutation im „Cystic Fibrosis Transmembrane Conductance Regulator" (CFTR)-Gen auf Chromosomen 7q13 ausgelöst. Bislang sind über 900 verschiedene Mutationen im CFTR-Gen identifiziert, die häufigste Mutation in Mitteleuropa ist die $\Delta$ F508-Mutation mit 75\% [1,2].

\section{Symptome}

Das typische klinische Erscheinungsbild des Mekoniumileus wird als Nixonsche Trias bezeichnet: Neugeborenenileus mit Erbrechen, geblähtem Abdomen, Stuhl- und Windverhaltung. Als Komplikationen kommen Volvolus, Perforation, Peritonitis und Atresie vor [3].

\section{Diagnostik}

Die Diagnose des Mekoniumileus wird anhand der Klinik und einer Röntgenübersichtsaufnahme sowie Ultraschalluntersuchung des Abdomens und eines Kolon-Kontrasteinlaufes gestellt.

\section{Differenzialdiagnose}

Als differenzialdiagnostische Überlegungen bei neonatalem Aszites sind die in Tab. $\mathbf{2}$ dargestellten Erkrankungen zu erwägen.

Tab. 2 Differenzialdiagnose bei neonatalem Aszites [4]

\begin{tabular}{|c|c|}
\hline \multicolumn{2}{|l|}{ Ursachen: } \\
\hline kardiovaskulär (20\%) & $\begin{array}{l}\text { Herzfehler } \\
\text { Arrhythmien } \\
\text { vaskuläre Anomalien }\end{array}$ \\
\hline Fehlbildungen (15\%) & $\begin{array}{l}\text { Skelett } \\
\text { Niere } \\
\text { Lunge } \\
\text { ZNS } \\
\text { gastrointestinal }\end{array}$ \\
\hline Chromosomenanomalien (15\%) & $\begin{array}{l}\text { Trisomie 13, 18, } 21 \\
\text { Turner-Syndrom }\end{array}$ \\
\hline Infektionen (5\%) & $\begin{array}{l}\text { CMV } \\
\text { Parvo-B19 } \\
\text { konnatale Hepatitis }\end{array}$ \\
\hline fetale Anämie (4\%) & fetofetalesTransfusionssyndrom \\
\hline $\begin{array}{l}\text { Tumoren (3\%) } \\
\text { metabolisch (1\%) }\end{array}$ & \\
\hline mütterlich (7\%) & $\begin{array}{l}\text { Diabetes mellitus } \\
\text { EPH-Gestose } \\
\text { Plazentafehlbildungen }\end{array}$ \\
\hline iopatisch (30\%) & \\
\hline
\end{tabular}

\section{Therapie}

Nur bei unkomplizierten Fällen kann ein konservativer Therapieversuch mit Gastrografineinlauf durchgeführt werden, um die Obstruktion zu lösen. In allen anderen Fällen ist eine frühzeitige Laparotomie nötig. Im günstigsten Fall kann eine primäre Anastomose, sonst eine doppelläufige lleostomie oder BischopKoop-Anastomose angelegt werden. Eine primäre Dünndarmresektion sollte nur dann durchgeführt werden, wenn ein Volvolus mit Darmgangrän, eine Perforation, eine Stenose oder Atresie vorliegen. Die Hemikolektomie ist in der Regel nicht nötig [3].

\section{Literatur}

1 Dockter G, Lindemann H. Mukoviszidose. Thieme 2000

2 Rosenstein BJ et al. The diagnosis of cystic fibrosis: a consensus statement. J Pediat 1998; 132: 589-595

${ }^{3}$ Voillital GM, Lehmann RR. Mekoniumileus. In: Chirurgie im Kindesalter. Spittau Verlag 2000; 288-293

${ }^{4}$ Byrne W. Disorders of the intestines and pancreas. In: Fanaroff AA, Martin RJ (eds). Neonatal-Perinatal Medicine: Diseases of the Fetus and Infant. Mosby 1997; $681-693$

\section{Dr. Simone Stoll-Becker}

Zentrum für Kinderheilkunde und Jugendmedizin Justus-Liebig-Universität Feulgenstraße 12 35392 Gießen 